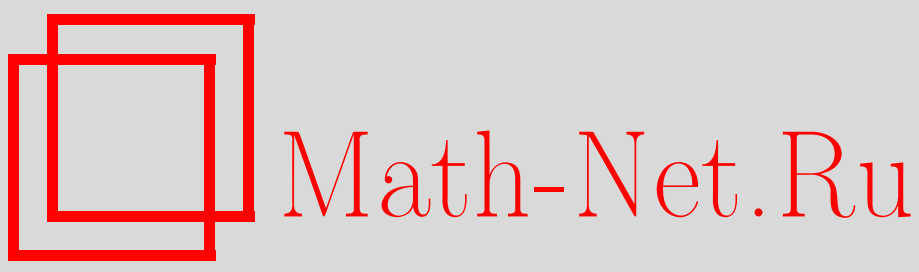

Н. Б. Енгибарян, Теоремы восстановления для системы интегральных уравнений, Матем. сб., 1998, том 189, номер 12, 59-72

DOI: https://doi.org/10.4213/sm360

Использование Общероссийского математического портала Math-Net.Ru подразумевает, что вы прочитали и согласны с пользовательским соглашением http://www . mathnet.ru/rus/agreement

Параметры загрузки:

IP : 54.147 .182 .235

26 апреля 2023 г., 03:58:52 
УДК $517.9+519.24$

\author{
Н. Б. Енгибарян
}

\title{
Теоремы восстановления для системы интегральных уравнений
}

Рассматривается система интегральных уравнений восстановления

$$
\varphi_{i}(x)=g_{i}(x)+\sum_{j=1}^{m} \int_{0}^{x} u_{i j}(x-t) \varphi_{j}(t) d t, \quad i=1, \ldots, m,
$$

где матрица-функция $u=\left(u_{i j}\right)$ удовлетворяет условиям консервативности $0 \leqslant u_{i j} \in L_{1}^{+} \equiv L_{1}(0 ; \infty)$, матрица $A=\int_{0}^{\infty} u(x) d x$ неразложимая и ее спектральный радиус равен 1 .

Доказано существование предела в $+\infty$ решения $\varphi=\left(\varphi_{1}, \ldots, \varphi_{m}\right)^{T}$ в случае, когда вектор-функция $g=\left(g_{1}, \ldots, g_{m}\right)^{T} \in L_{1}^{m}$ ограничена и $g(+\infty)=0$. Выгислен этот предел. Найдена структура $\varphi$ при $g \in L_{1}^{m}: \varphi(x)=\mu+\rho_{0}(x)+$ $\psi(x)$, где $\rho_{0} \in C_{0}^{m}, \psi \in L_{1}^{m}$. Получена аналогичная формула для резольвентной матрицы-фоункции.

Библиографияя: 15 названий.

\section{Введение}

В теории вероятностей и в математической физике важную роль играют скалярные и векторные интегральные Уравнения Восстановления (УВ) (см. [1]-[11]):

$$
\varphi(x)=g(x)+\int_{0}^{x} u(x-t) \varphi(t) d t .
$$

В скалярном случае $u$ является плотностью распределения неотрицательной случайной величины:

$$
0 \leqslant u \in L_{1} \equiv L_{1}(0 ; \infty), \quad \int_{0}^{\infty} u(x) d x=1 .
$$

В векторном случае (0.1) представляет собой векторно-матричную запись следуюшей системы УВ:

$$
\varphi_{i}(x)=g_{i}(x)+\sum_{j=1}^{m} \int_{0}^{x} u_{i j}(x-t) \varphi_{j}(t) d t, \quad i=1, \ldots, m .
$$

Естественным аналогом условий (0.2) в случае системы (0.3) являются следующие условия критичности или консервативности (см. [2]-[11]):

$$
u=\left(u_{i j}\right) \geqslant 0 \quad \text { (покомпонентно) }, \quad A \equiv \int_{0}^{\infty} u(x) d x \in P_{N}, \quad \lambda=1 .
$$


Здесь $P_{N}-$ класс неотрицательных неразложимых (неприводимых $)(m \times m)$-матриц, $\lambda=r(A)$ - спектральный радиус матрицы $A$ (см. [12]). В теории вероятностей уравнения (0.3) называют уравнениями многомерного восстановления (УМВ). Важньм частным случаем УМВ являются уравнения марковского восстановления (тогда матрица $A$ является стохастической). УМВ возникают в теории массового обслуживания, теории надежности, исследовании операций, теории ветвящихся процессов и др. (см. [4]-[6]). УМВ, кроме их самостоятельного значения, представляют интерес также как результат факторизации систем интегральных уравнений Винера-Хопфа (см. [8]-[10]).

Существование и единственность решения скалярного и векторного уравнения $(0.1)$ в классе локальных интегрируемых на $[0 ; \infty)$ функций хорошо известна (см. [2], [10]). Вопрос асимптотического поведения решения $\varphi$ УВ в критическом случае относится к главным задачам теории УВ. Одной из основных задач классической теории восстановления (в теории вероятностей) является вопрос существования и вычисления усредненного или обычного предела $\varphi$ в $+\infty$ в случае скалярного УВ (см. [1], [2]). Решение этой задачи дается теоремами восстановления Блекуэлла, Смита и их различньми обобщениями. Эта задача в настояшее время достаточно хорошо изучена (см. [1], [2], [6], [13]). Теория восстановления для УМВ развита менее полно (см. [2]-[11]).

Приведем некоторые известные результаты по асимптотическим свойствам решения УМВ.

В работе З.Б. Цалюка [3] рассмотрена система $(0.3),(0.4)$ в случае, когда $\int_{0}^{x} u(t) d t<A \quad \forall x<+\infty$ (следовательно, $A$ - строго положительна), $g$ и $u$ непрерывны на $[0 ; \infty)$ и $\int_{0}^{x} t u(t) d t=(C+o(1)) x^{\alpha}, 0<\alpha<1$. Доказано, что тогда из сушествования предела $g(+\infty)$ следует сушествование предела $x^{-\alpha} \varphi(x)$ в $+\infty$. Вычислен этот предел. В работе Б. А. Севастьянова и В.П. Чистякова [4] изучены асимптотические свойства решения критического УМВ в предположении конечности второго или третьего моментов матрицы-функции $u$. В [5] приведены две теоремы восстановления для одного частного случая критического УМВ. Предполагается, что рассматриваемое уравнение порождается неприводимым возвратным полумарковским процессом. Об этих теоремах речь пойдет в конце настоящей работы.

Данная работа посвящена новым теоремам восстановления для УВМ в критическом случае (0.4). Развиваемый подход основан на результатах работы [13] и на гауссовской треугольной факторизации матричной структуры уравнения (0.3) методом работы автора [14].

\section{§1. Вспомогательные предложения}

1.1. Пусть $\mathbb{R}^{m}$ и $\mathbb{R}_{m}$ - пространства вешественных $m$-мерных вектор-столбцов и вектор-строк, соответственно; $\mathbb{R}^{m \times m}$ - алгебра $(m \times m)$-матриц с единицей $I$. Через $A^{T}$ обозначается матрица или вектор, транспонированный к $A$. Записи $A>0$ и $A \geqslant 0$ означают, что все элементы $A$ положительны или неотрицательны, $A \succ B$ означает, что $A \geqslant B$ и $A \neq B$. Пусть $P_{N} \subset \mathbb{R}^{m \times m}$ - класс неотрицательных неразложимых матрищ (см. [12]). Через $r(A)$ обозначается спектраль- 
ный радиус матрицы $A$, т.е. модуль наибольшего по модулю собственного значения $A \in \mathbb{R}^{m \times m}$.

Теорема Перрона-Фробениуса (см. [12; теорема 9.2.1]). Ecли $A \in P_{N}, m o$ A имеет положстельное собственное значение $\lambda$, равное спектральному радиусу $r(A)$. Существует положсительный правый собственный вектор $\eta$, соответствующий собственному значению $\lambda$. Собственное значение $\lambda$ имеет алгебраическую кратность 1 .

Так как из $A \in P_{N}$ следует $A^{T} \in P_{N}$, то собственному значению $\lambda$ соответствует также левый собственньй вектор $\zeta \in \mathbb{R}_{m}$.

Поэтому имеют место равенства

$$
A \eta=\lambda \eta, \quad \zeta A=\lambda \zeta
$$

где

$$
\lambda=r(A), \quad \eta \in \mathbb{R}^{m}, \quad \eta>0, \quad \zeta \in \mathbb{R}_{m}, \quad \zeta>0 .
$$

1.2. Функциональные пространства. введем в рассмотрение следующие банаховы пространства (БП) определенных на [0; $\infty)$ функций: $L_{p}, 1 \leqslant p<+\infty$, $M, M_{u}, M_{0}, C_{M}, C_{u}, C_{0}$. Здесь $M$ - пространство ограниченных в сушественном функций, $C_{M} \subset M-$ пространство непрерывных и ограниченных на $\mathbb{R}^{+}$функций, $M_{u} \subset M$ и $C_{u} \subset C_{M}$ состоят из функций $f$, имеющих конечньй предел $f(+\infty)$ в $+\infty$. Если $f \in M_{0} \subset M_{u}$ или $f \in C_{0} \subset C_{u}$, то $f(+\infty)=0$. Пусть $E-$ одно из банаховых пространств, перечисленных выше.

Обозначим через $E^{m}$ пространство $m$-мерных вектор-столбцов с элементами из $E$, через $E^{m \times m}-$ пространство $(m \times m)$-матриц-функций с элементами из $E$. Пространство $L_{1}^{m \times m}$ является сверточной банаховой алгеброй с умножением

$$
(u * v)(x)=\int_{0}^{x} u(x-t) v(t) d t, \quad u, v \in L_{1}^{m \times m} .
$$

Хорошо известные свойства свертки мы здесь не приводим, используемые свойства операторов свертки сформулируем в тексте изложения.

1.3. Результаты [13] по скалярным УВ (0.1), (0.2) приведем в виде следующей теоремы.

Tеорема А. а) Пусть в системе (0.1), (0.2) $g \in L_{1} \cap M_{0}$. Тогда $\varphi \in M_{u} u$

$$
\varphi(+\infty) \equiv \mu=\nu^{-1} \int_{0}^{\infty} g(x) d x, \quad \nu^{-1} \geqslant 0,
$$

$2 \partial e$

$$
\nu=\int_{0}^{\infty} x u(x) d x \leqslant+\infty
$$

б) $\operatorname{Ecлu~} g \in L_{1}$, mo

$$
\varphi(x)=\mu+\rho(x)+\psi(x)
$$

əде $\rho \in C_{0}, \psi \in L_{1}$.

Теорема восстановления (альтернативная форма [1, гл. XI, § I]) утверждает выполнение предельного соотношения (1.2) в случае, когда функция $g$ непосредственно интегрируема по Риману на $\mathbb{R}^{+}$(ясно, что тогда $g \in L_{1} \cap M_{0}$ ). 


\section{§2. Факторизация матрицы $I-A$}

Изучение системы (0.3), (0.4) связано с рассмотрением вспомогательной задачи треугольной факторизации необратимой матрицы $I-A$, где $A$ - матрица, фигурируюшая в (0.4).

Введем подалгебры $G^{+}$и $C^{-}$алгебры $\mathbb{R}^{m \times m}$ : если $B=\left(b_{i j}\right) \in G^{+}$, то $b_{i j}=0$ при $j \geqslant i$; если $C=\left(c_{i j}\right) \in G^{-}$, то $c_{i j}=0$ при $j<i$.

Имеем $\mathbb{R}^{m \times m}=G^{+} \oplus G^{-}$. Пусть $P_{+}$и $P_{-}-$проекторы, отображающие $\mathbb{R}^{m \times m}$ в $G^{+}$и $G^{-}$, соответственно.

Рассмотрим задачу построения факторизации

$$
I-A=(I-B)(I-C)
$$

где $B \in G^{+}$и $C \in G^{-}$- искомые матрицы. Из (2.1) имеем $B+C=A+B C$, откуда

$$
\begin{aligned}
& B=A^{+}+P_{+}(B C), \\
& C=A^{-}+P_{-}(B C),
\end{aligned}
$$

где $A^{ \pm}=P_{ \pm} A, A^{+}+A^{-}=A$.

Соотношения (2.2) в раскрытом виде представляют собой хорошо известные уравнения, возникающие при факторизационной трактовке метода последовательных исключений Гаусса в линейной алгебре. Методом работы [8] не только доказывается существование факторизации (2.1) необратимой матрицы $I-A$, но и получается нужная для дальнейшего изложения информация о свойствах матриц $B$ и $C$.

Пусть $\eta \in \mathbb{R}^{m}, \eta>0$ и $\zeta \in \mathbb{R}_{m}, \zeta>0$ суть суть правосторонний и левосторонний неподвижные векторы матрицы $A$, соответственно. Их существование следует из (0.4) и теоремы Перрона-Фробениуса (см. §1). Имеем

$$
A \eta=\eta, \quad \eta>0, \quad \zeta A=\zeta, \quad \zeta>0 .
$$

Рассмотрим следующие последовательные приближения для (2.2):

$$
\begin{gathered}
B_{n+1}=A^{+}+P_{+}\left(B_{n} C_{n}\right), \quad C_{n+1}=A^{-}+P_{-}\left(B_{n} C_{n}\right), \\
B_{0}=C_{0}=0, \quad n=0,1, \ldots
\end{gathered}
$$

Индукцией по $n$ легко проверяется, что соотношения (2.4) определяют последовательности неотрицательных матриц $B_{n}=\left(b_{i j}^{(n)}\right)$ и $C_{n}=\left(c_{i j}^{(n)}\right)$, которые покомпонентно монотонно возрастают по $n$. Из (2.4) имеем

$$
B_{n+1} \eta=A^{+} \eta+P_{+}\left(B_{n} C_{n}\right) \eta, \quad C_{n+1} \eta=A^{-} \eta+P_{-}\left(B_{n} C_{n}\right) \eta,
$$

складывая которые с учетом $A \eta=\eta$ получаем

$$
B_{n+1} \eta+C_{n+1} \eta=\eta+B_{n} C_{n} \eta .
$$


Докажем индукцией по $n$ неравенство (для $n=0$ оно тривиально)

$$
C_{n} \eta \leqslant \eta
$$

Пусть (2.6) выполняется для некоторого $n \geqslant 0$. Из (2.5) имеем

$$
C_{n+1} \eta=\eta-\left(B_{n+1}-B_{n}\right) \eta-B_{n}\left(\eta-C_{n} \eta\right)
$$

Так как $B_{n+1} \geqslant B_{n}$ и $\eta-C_{n} \eta \geqslant 0$, получаем $C_{n+1} \eta \leqslant \eta$.

Аналогично доказывается неравенство

$$
\zeta B_{n} \leqslant \zeta
$$

Из (2.6) и (2.7) следуют оценки для элементов матриц $B_{n}$ и $C_{n}$ :

$$
b_{i j}^{(n)} \leqslant \frac{\zeta_{j}}{\zeta_{i}}, \quad c_{i j}^{(n)} \leqslant \frac{\eta_{i}}{\eta_{j}} .
$$

Последовательности $\left(b_{i j}^{(n)}\right)_{n=0}^{\infty}$ и $\left(c_{i j}^{(n)}\right)_{n=0}^{\infty}$ монотонно возрастают и ограничены сверху, поэтому они сходятся, т.е. сушествуют матрицы $B \in G^{+}$и $C \in G^{-}$такие, что $B_{n} \uparrow B$ и $C_{n} \uparrow C$ покомпонентно. Совершая в $(2.4),(2.6)$ и (2.7) предельный переход при $n \rightarrow+\infty$ получаем, что $(B, C)$ удовлетворяет соотношениям $(2.2)$ и имеют место неравенства

$$
C \eta \leqslant \eta, \quad \zeta B \leqslant \zeta
$$

Нами доказано существование факторизации (2.1). Рассмотрим теперь следующий вопрос: в каком из неравенств (2.8) имеет место равенство? Так как диагональные элементы треугольной матрицы $B$ равны 0 , то $B^{m}=0$, т.е. $B$ - нильпотентная матрица с индексом $\leqslant m-1$ (см. [15]). Поэтому матрица $I-B$ обладает положительной обратной вида $(I-B)^{-1}=I+\widetilde{B}, \widetilde{B}=B+\cdots+B^{m-1} \in G^{+}$, $\zeta-\zeta B \succ 0$. Из равенства $A \eta=\eta$ с учетом факторизации $(2.1)$ и обратимости $I-B$ имеем $C \eta=\eta$.

Рассмотрим диагональные элементы матрицы $C$. Перепишем равенство $C \eta=\eta$ в раскрытом виде:

$$
\eta_{i}=\sum_{j=i}^{m} c_{i j} \eta_{j}, \quad i=1, \ldots, m .
$$

При $i=m$ получаем $\eta_{m}=c_{m m} \eta_{m}$. Так как $\eta_{m}>0$, то $c_{m m}=1$. Покажем, что $c_{k k}<1$ при $k<m$, т.е. $c_{m m}$ - единственный диагональный элемент матрицы $C$, равньй 1. Из равенств (2.9) имеем $\left(1-c_{k k}\right) \eta_{k}=\sum_{j=k+1}^{m} c_{k j} \eta_{j} \geqslant 0$. Поэтому $c_{k k} \leqslant 1$. Пусть $c_{k k}=1$ при некотором $k<m$. Будем считать, что $k-$ наименњший из номеров $i$, при котором $c_{i i}=1$. Рассмотрим однородное уравнение $C \tau=\tau$. В силу $c_{k k}=1$ и $c_{i i}<1$ при $i<k$ это уравнение имеет нетривиальное решение вида $\tau=\left(\tau_{1}, \ldots, \tau_{k-1}, 1,0, \ldots, 0\right)^{T}$, где $\tau_{1}, \ldots, \tau_{k-1}$ определяются из следующей треугольной системы с обратимой матрицей:

$$
\tau_{i}=c_{i k}+\sum_{j=i}^{k-1} c_{i j} \tau_{j}, \quad i=1, \ldots, k-1 .
$$


Из $(I-C) \tau=0$ в силу $(2.1)$ имеем $(I-A) \tau=0$. Мы получили, что матрица $A \in P_{N}$ обладает вторым неподвижным вектором $\tau \in \mathbb{R}^{m}$, линейно не зависящим от $\eta$, что противоречит теореме Перрона-Фробениуса (см. $\mathrm{I})$. Поэтому $c_{k k}<1$ при $k<m$.

Рассмотрим вектор-строку $\gamma=\zeta(I-B) \succ 0$. Из равенства $\zeta(I-A)=0$ и факторизации (2.1) следует $\gamma(I-C)=0$. В силу треугольности матрицы $C$ и вьполнения неравенств $c_{k k}<1$ при $k<m$ имеем $\gamma_{1}=\gamma_{2}=\cdots=\gamma_{m-1}=0$. Из равенства $\gamma=\zeta(I-B)$ и $B \in G^{+}$легко следует $\gamma_{m}=\zeta_{m}$. Поэтому

$$
\gamma \equiv \zeta-\zeta B=\left(0,0, \ldots, 0, \zeta_{m}\right)
$$

Нами доказана

Теорема 2.1. Пусть матрица $A \in \mathbb{R}^{m \times m}$ удовлетворяет условиям

$$
A \in P_{N}, \quad r(A)=1 \text {. }
$$

Тогда имеет место факторизачия (2.1), где треугольные матрицы $0 \leqslant B$ $\in G^{+}, 0 \leqslant C \in G^{-}$обладают следующими свойствами ( и и удовлетворяют (2.3)): $c_{m m}=1, c_{k k}<1$ nрu $k<m, C \eta=\eta, \zeta-\zeta B=\left(0, \ldots, 0, \zeta_{m}\right)$.

\section{§ 3. Факторизация уравнения (0.3) с условием $(0.4)$}

В настоящем параграфе факторизация (2.1) будет использована для факторизации матричной структуры системы $(0.3)$, (0.4). Перепишем векторное уравнение $(0.1)$, т.е. уравнение $(0.3)$, в операторном виде:

$$
(J-U) \varphi=g
$$

Здесь $J$ - единичньй оператор, действующий в пространстве $E^{m}$, а $U$ - интегральный оператор, ограниченный в $E^{m}$ (см. [13], [10]),

$$
(U \varphi)(x)=\int_{0}^{x} u(x-t) \varphi(t) d t, \quad u \in L_{1}^{m \times m}
$$

Пусть $\Omega$ - алгебра матричных интегральных операторов вида (3.2). Обозначим через $\Omega^{+}$и $\Omega^{-}$следуюшие подалгебры $\Omega$ :

если $V \in \Omega^{+}$, то $v_{i j}(x)=0, j \geqslant i$;

если $W \in \Omega^{-}$, то $w_{i j}(x)=0, j<i$.

Имеем $\Omega=\Omega^{+} \oplus \Omega^{-}$.

Рассмотрим следуюшую задачу факторизации: для $U \in \Omega$ найти $V \in \Omega^{+}$и $W \in \Omega^{-}$такие, что

$$
J-U=(J-V)(J-W)
$$

Из (3.3) получаем следуюшее соотношение между ядрами:

$$
v(x)+w(x)=u(x)+\int_{0}^{x} v(x-t) w(t) d t
$$


Применяя к (3.4) проекторы $P_{+}$и $P_{-}$получаем

$$
\begin{gathered}
v(x)=u_{+}(x)+P_{+}\left(\int_{0}^{x} v(x-t) w(t) d t\right), \\
w(x)=u_{-}(x)+P_{-}\left(\int_{0}^{x} v(x-t) w(t) d t\right),
\end{gathered}
$$

где $u_{ \pm}(x)=P_{ \pm}(u(x))$.

Соотношения (3.5) представляют собой уравнения треугольной факторизации в рамках метода Гаусса в том случае, когда элементами матриц служат элементы сверточной алгебры $L_{1}^{m \times m}$.

Для изучения нелинейной системы (3.5) аналогично $\S 2$ рассмотрим последовательные приближения:

$$
\begin{gathered}
v_{n+1}(x)=u_{+}(x)+P_{+}\left(\int_{0}^{x} v_{n}(x-t) w_{n}(t) d t\right) \\
w_{n+1}(x)=u_{-}(x)+P_{-}\left(\int_{0}^{x} v_{n}(x-t) w_{n}(t) d t\right) \\
v_{0}=w_{0}=0, \quad n=0,1, \ldots
\end{gathered}
$$

Ясно, что соотношения (3.6) определяют последовательности матриц-функций $v_{n} \in \Omega^{+}$и $w_{n} \in \Omega^{-}$с неотрицательными элементами из $L_{1} \quad v_{n}=\left(v_{i j}^{(n)}\right)$, $w_{n}=\left(w_{i j}^{(n)}\right)$, причем $v_{i j}^{(n)}$ и $w_{i j}^{(n)}$ возрастают по $n$.

Обозначим

$$
B_{n}=\int_{0}^{\infty} v_{n}(x) d x, \quad C_{n}=\int_{0}^{\infty} w_{n}(x) d x
$$

Интегрируя соотношения (3.6) по $x$ от 0 до $+\infty$, приходим к соотношениям (2.4). Из свойств итераций (2.4), установленных в $\S 2$, следует, что возрастающие последовательности неотрицательных функций $v_{i j}^{(n)}$ и $w_{i j}^{(n)}$ ограничены по норме пространства $L_{1}$ числами $b_{i j}$ и $c_{i j}$, соответственно. Поэтому согласно теореме Леви [11] эти функциональные последовательности сходятся в $L_{1}$ :

$$
v_{i j}^{(n)} \uparrow v_{i j} \in L_{1}, \quad w_{i j}^{(n)} \uparrow w_{i j} \in L_{1}
$$

Пусть $v=\left(v_{i j}\right), w=\left(w_{i j}\right)$. Имеем

$$
v \geqslant 0, \quad w \geqslant 0, \quad \int_{0}^{\infty} v(x) d x=B \equiv\left(b_{i j}\right), \quad \int_{0}^{\infty} w(x) d x=C \equiv\left(c_{i j}\right) .
$$

Законность предельного перехода в соотношениях (3.6) следует из непрерывной зависимости свертки (1.1) от $u$ и $v$. Справедлива

ТЕОРема 3.1. В консервативном случае (0.4) оператор $J-U$ допускает факторизачию (3.3), әде $V \in \Omega^{+}, W \in \Omega^{-}$. Имеют место равенства (3.7), В и С обладают свойствами, перечисленными в теореме 2.1 . 


\section{$\S 4$. Существование предела $\varphi(+\infty)$ в векторном случае}

В качестве применения факторизации (3.3) мы будем рассматривать вопрос существования и выгисления предела $\varphi(+\infty)$ в случае векторного уравнения $(0.1)$ с условием (0.4).

С учетом (3.3) уравнение (0.1) можно переписать в виде

$$
(J-V)(J-W) \varphi=g .
$$

Обозначим

$$
(J-W) \varphi=f,
$$

$f$ удовлетворяет уравнению

$$
(J-V) f=g .
$$

Так как матричное ядро $v$ принимает значения из $G^{+}$, то оператор $V$ - нильпотентньй с индексом $\leqslant m-1$. Поэтому

$$
(J-V)^{-1}=J+\widetilde{V}, \text { где } \widetilde{V}=V+V^{2}+\cdots+V^{m-1} \in \Omega^{+} .
$$

Согласно (4.4) решение уравнения (4.3) имеет вид:

$$
f=g+\widetilde{V} g, \quad \tilde{V} \in G^{+}, \quad \tilde{v} \geqslant 0 .
$$

Из (4.5) видно, что

a) если $g \in E^{m}$, то $f \in E^{m}$;

б) если $g \geqslant 0$, то $f \geqslant 0$.

Рассмотрим теперь уравнение (4.2) относительно $\varphi$. Перепишем его в раскрытом виде

$$
\varphi_{i}(x)=f_{i}(x)+\sum_{j=i}^{m} \int_{0}^{x} w_{i j}(x-t) \varphi_{j}(t) d t .
$$

Рассмотрим последнее из уравнений $(4.6)$ (когда $i=m)$ :

$$
\varphi_{m}(x)=f_{m}(x)+\int_{0}^{x} w_{m m}(x-t) \varphi_{m}(t) d t .
$$

Из (3.7) и (2.16) следует, что скалярное уравнение (4.7) удовлетворяет условиям консервативности (0.2). Поэтому для этого уравнения справедливы утверждения теоремы А из $\S 1$. Если $g \in L_{1}^{m} \cap M_{0}^{m}$, то $f \in L_{1}^{m} \cap M_{0}^{m}$ и $\varphi_{m} \in M_{u}$. Если же $g \in L_{1}^{m}$, то $f \in L_{1}^{m}$ и функция $\varphi_{m}$ имеет вид $\varphi_{m}(x)=\rho_{m}(x)+\psi_{m}(x)$, где $\rho_{m} \in C_{u}$ и $\psi_{m} \in L_{1}$.

Подставляя найденную функцию $\varphi_{m}$ в уравнение $(4.6)$ при $i=1, \ldots,(m-1)$, получаем

$$
\varphi_{i}(x)=F_{i}(x)+\sum_{j=i+1}^{m-1} \int_{0}^{x} w_{i j}(x-t) \varphi_{j}(t) d t+\int_{0}^{x} w_{i i}(x-t) \varphi_{i}(t) d t
$$


где

$$
F_{i}(x)=f_{i}(x)+\int_{0}^{x} w_{i m}(x-t) \varphi_{m}(t) d t
$$

Если $g \in L_{1} \cap M_{0}$, то $f_{i} \in M_{0}, F_{i} \in M_{u}$. Из неравенств (2.16) следует, что все операторы $W_{i i}$ с ядрами $w_{i i}$ - сжимающие в $E$, так как $\left\|W_{i j}\right\|_{E} \leqslant \int_{0}^{\infty}\left|w_{i j}(x)\right| d x$. Поэтому операторы $J-W_{i i}, i=1, \ldots,(m-1)$, обладают положительными обратными. Тогда из уравнений (4.8) можно рекуррентно по $k$ определить $\varphi_{m-k} \in M_{u}$, $k=1, \ldots,(m-1)$. В результате строится решение $\varphi \in M_{u}^{m}$ уравнения (4.2), а следовательно, - и решение исходной системы $(0.1),(0.4)$. Аналогичным образом можно построить решение системы (4.8) в случае $g \in L_{1}^{m}$. Тогда будем иметь $\varphi_{i}=\rho_{i}+\psi_{i}$, где $\rho_{i} \in C_{0}, \psi_{i} \in L_{1}, i=1, \ldots, m$.

Нами доказана

Tеорема 4.1. а) Пусть в системе (0.1), (0.4) $g \in L_{1}^{m} \cap M_{0}^{m}$. Тогда $\varphi \in M_{u}^{m}$. б) Если $g \in L_{1}^{m}$, то решение (0.1), (0.4) имеет вид:

$$
\varphi=\rho+\psi, \quad \rho \in C_{u}^{m}, \quad \psi \in L_{1}^{m}
$$

\section{§5. Теоремы восстановления для уравнения (0.1) с условием $(0.4)$}

Предел в $+\infty$ вектор-функции $\rho$, фигурирующей в представлении (4.10), может быть вычислен, исходя из формулы (2.1) для скалярного уравнения (4.7) и конструкций предыдущего параграфа. Однако этот путь достаточно трудоемкий и может быть заменен более простой процедурой, которая будет изложена ниже.

ЛЕмма 5.1. Вектор-функиия $\rho \in C_{u}^{m}$, фигурирующая в (4.10), имеет следующий предел в $+\infty$ :

$$
\rho(+\infty)=\mu \equiv(\zeta \nu \eta)^{-1}\left(\zeta \int_{0}^{\infty} g(x) d x\right) \eta
$$

где $\eta \in \mathbb{R}^{m}$ и $\zeta \in \mathbb{R}_{m}$ суть положительные, правый и левыи, неподвижные векторьи матрицы $A$, а матрица $\nu$ - первыи момент матрицы-функици и :

$$
\nu=\int_{0}^{\infty} x u(x) d x
$$

Если $\nu<+\infty \quad\left(\right.$ покомпактно), то число $\varkappa \equiv(\zeta \nu \eta)^{-1}>0$. Если же хотя бъ одна компонента р равна $+\infty$, то $\varkappa=0, \mu=0$. 
ДокАЗАТЕЛЬСТво. Пусть $\mu^{\prime}=\rho(+\infty)$ и $\rho_{0}(x)=\rho(x)-\mu^{\prime}$. Из $(0.1)$ получаем

$$
(I-A) \mu^{\prime}=\sigma_{1}(x)+\sigma_{2}(x),
$$

где

$$
\begin{aligned}
& \sigma_{1}(x)=g(x)+\int_{0}^{x} u(x-t) \psi(t) d t-\psi(x) \\
& \sigma_{2}(x)=-\rho_{0}(x)-\left(\int_{x}^{\infty} u(t) d t\right) \mu^{\prime}+\int_{0}^{x} u(x-t) \rho_{0}(t) d t .
\end{aligned}
$$

Имеем $\sigma_{1} \in L_{1}^{m}, \sigma_{2} \in C_{0}^{m}$.

Равенство (5.3) возможно только лишш в том случае, когда $(I-A) \mu^{\prime}=0$. В силу $A \in P_{N}, r(A)=1$ и $A \eta=\eta$ согласно теореме Перрона-Фробениуса имеем

$$
\mu^{\prime}=\lambda_{0} \eta, \quad \lambda_{0} \in \mathbb{R}
$$

Остается найти значение числа $\lambda_{0}$. Применяя к (0.1) преобразование Лапласа (ПЛ), получаем

$$
(I-\bar{u}(s))\left(\bar{\rho}_{0}(s)+\bar{\psi}(s)+\frac{\lambda_{0}}{s} \eta\right)=\bar{g}(s) .
$$

Через $\bar{f}(s)$ обозначается ПЛ (скалярной или матричной) функции $f$ :

$$
\bar{f}(s)=\int_{0}^{\infty} f(x) e^{-s x} d x, \quad s>0 .
$$

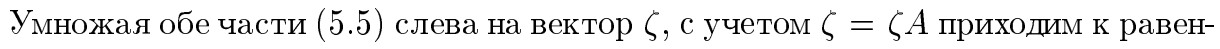
ству

$$
\frac{1}{s} \zeta(A-\bar{u}(s))\left(s \bar{\rho}_{0}(s)+s \bar{\psi}(s)+\lambda_{0} \eta\right)=\bar{g}(s) .
$$

Из $\psi \in L_{1}^{m}$ следует ограниченность $\bar{\psi}(s)$ в окрестности $s=0$. Поведение $s \bar{\rho}_{0}(s)$ в окрестности $s=0$ определяется следуюшей элементарной теоремой Абелева типа: если $f \in M_{0}$, то $\bar{f}(s)=o(1 / s)$ при $s \rightarrow 0+$. Из сказанного следует

$$
s \bar{\rho}_{0}(s)+s \bar{\psi}(s) \rightarrow 0 \text { при } s \rightarrow 0+.
$$

Так как $A=\bar{u}(0)$, то

$$
\lim _{s \rightarrow 0+} \frac{1}{s}(A-\bar{u}(s))=\nu \equiv \int_{0}^{\infty} x u(x) d x .
$$

Некоторые компоненты $\nu$ могут быть равны $+\infty$. Сначала рассмотрим случай $\nu<+\infty$. Совершая в (5.6) предельный переход при $s \rightarrow 0+$, с учетом $(5.7)$ и $(5.8)$ получаем

$$
\lambda_{0} \zeta \nu \eta=\zeta \bar{g}(0), \quad \lambda_{0}=(\zeta \nu \eta)^{-1} \zeta \int_{0}^{\infty} g(x) d x,
$$

откуда приходим к формуле (5.1). 
Пусть теперь хотя бы одна компонента матрицы $\nu$ равна $+\infty$. Пусть $\nu_{k p}=+\infty$. Обозначим

$$
Q(s)=\frac{1}{s}(A-\bar{u}(s)), \quad \omega(s)=\zeta Q(s)=\left(\omega_{1}(s), \ldots, \omega_{m}(s)\right) .
$$

Имеем $Q_{i j}(s) \geqslant 0, \zeta>0$ и $Q_{k p}(s) \rightarrow+\infty$ при $s \rightarrow 0+$. Из неравенства $\omega_{p}(s) \geqslant \zeta_{k} Q_{k p}(s)$ следует

$$
\omega_{p}(s) \rightarrow+\infty \text { и } \omega(s) \eta \rightarrow+\infty \text { при } s \rightarrow 0+.
$$

Докажем равенство $\lambda_{0}=0$ от противного. Пусть $\lambda_{0} \neq 0$. Тогда из (5.6) будем иметь

$$
\omega(s)\left(\eta-\frac{1}{\lambda_{0}} s \bar{\rho}(s)-\frac{1}{\lambda} s \bar{\psi}(s)\right)=-\frac{1}{\lambda_{0}} \zeta \bar{g}(s) .
$$

При $s \rightarrow 0+$ правая часть этого равенства имеет конечный предел, а левая часть стремится к $+\infty$. Из полученного противоречия следует $\lambda_{0}=0$. Так как $\zeta \nu \eta=+\infty$ при $\nu_{k p}=+\infty$, то равенство $\lambda_{0}=0$ означает, что формула (5.1) остается в силе, когда некоторые компоненты $\nu$ равны $+\infty$. Лемма доказана.

В условиях а) и б) теоремы 4.1 имеем $g \in L_{1}^{m}$ и сушествует решение системы $(0.1),(0.4)$ в виде (4.10). Поэтому в условиях теоремы 4.1 может быть применена лемма 5.1. В результате мы приходим к следующей теореме восстановления.

ТЕОРема 5.1 (теорема восстановления). а) Пусть в уравнении (0.1) с условием (0.4) $g \in L_{1}^{m}$. Тогда решение системь имеет вид:

$$
\varphi(x)=\mu+\rho_{0}(x)+\psi(x),
$$

где $\rho_{0} \in C_{0}^{m}, \psi \in L_{1}^{m}$, а вектор $\mu \in \mathbb{R}^{m}$ определяется по (5.1).

б) Ecли $g \in L_{1}^{m} \cap M_{0}^{m}$, mo $\psi \in L_{1}^{m} \cap M_{0}^{m} u$

$$
\varphi(+\infty)=(\zeta \nu \eta)^{-1}\left(\zeta \int_{0}^{\infty} g(x) d x\right) \eta, \quad(\zeta \nu \eta)^{-1} \geqslant 0 .
$$

\section{§6. Основная теорема восстановления}

Настоящий параграф посвящен доказательству теоремы восстановления для резольвентной матрицы-функции уравнения (0.1) с условием (0.4).

6.1. Пусть $\Phi$ резольвентная матрица-функция рассматриваемой системы уравнений. Она определяется из УВ

$$
\Phi(x)=u(x)+\int_{0}^{x} u(x-t) \Phi(t) d t .
$$

Решение уравнения (0.1) выражается через $\Phi$ по формуле (см. [4]):

$$
\varphi(x)=g(x)+\int_{0}^{x} \Phi(x-t) g(t) d t,
$$


$k$-й столбец $\Phi_{k}$ матрицы-функции $\Phi$ удовлетворяет векторному уравнению

$$
\Phi_{k}(x)=u_{k}(x)+\int_{0}^{x} u(x-t) \Phi_{k}(t) d t
$$

где $u_{k}-k$-й столбец $u$. Согласно теореме восстановления 5.1 имеем

$$
\Phi_{k}(x)=\mu_{k}+\rho_{k}^{0}(x)+\psi_{k}(x), \quad k=1, \ldots, m,
$$

где $\rho_{k}^{0} \in C_{0}^{m}, \psi_{k} \in L_{1}^{m}$. Для $\mu_{k}$ получается выражение

$$
\mu_{k}=(\zeta \nu \eta)^{-1}\left(\zeta A_{k}\right) \eta
$$

Из равенства $\zeta A=\zeta$ получаем $\zeta A_{k}=\zeta_{k}$, где $\zeta_{k}-k$-я компонента $\zeta$. С учетом этого из (6.4) и (6.5) получаем

$$
\Phi(x)=H+F(x)+\Psi(x),
$$

где $H=\left(\mu_{1}, \ldots, \mu_{m}\right)$ - матрица, $k$-м столбцом которой служит $\mu_{k}$,

$$
F=\left(\rho_{1}^{0}, \ldots, \rho_{m}^{0}\right) \in C_{0}^{m \times m}, \quad \Psi=\left(\psi_{1}, \ldots, \psi_{m}\right) \in L_{1}^{m \times m} .
$$

Нетрудно проверить, что

$$
H=(\zeta \nu \eta)^{-1} \eta \zeta, \quad(\zeta \nu \eta)^{-1} \geqslant 0 .
$$

Интегрируя (6.6) по $x$ от $t$ до $t+h$, где $t, h>0$, получаем

$$
\int_{t}^{t+h} \Phi(x) d x=h H+\int_{t}^{t+h}(F(x)+\Psi(x)) d x .
$$

Из $F \in C_{0}^{m \times m}$ и $\Psi \in L_{1}^{m \times m}$ следует, что интеграл в правой части (6.8) стремится к 0 при $t \rightarrow+\infty$. Совершая в (6.8) предельный переход при $t \rightarrow+\infty$, получаем

$$
\lim _{t \rightarrow+\infty} \int_{t}^{t+h} \Phi(x) d x=h \cdot H \quad \forall h>0 .
$$

Нами доказана

Теорема 6.1 (теорема восстановления, основная форма). а) Резольвентная матрица-функция $\Phi$ векторного уравнения (0.1) с условием (0.4) имеет структуру (6.6), где $F \in C_{0}^{m \times m}, \Psi \in L_{1}^{m \times m}$, а Н определяется согласно (6.7).

б) Имеет место предельное соотночение (6.9).

ЗАмечАнИЕ. Из формулы (6.6) следует не только (6.9), но теорема 5.1.

6.2. В настояшем пункте будут сделаны некоторые замечания по полученным результатам и их возможным обобшениям.

Прежде всего сформулируем две теоремы восстановления, изложенные в $\S 3$ обзорной работы [5] (формулы (3.2) и (3.3)). Об этих теоремах шла речь во введении. В обозначениях, принятых в настоящей работе, эти теоремы в случае абсолютно непрерывного распределения могут быть сформулированы в следуюшем виде. 
ТЕоремА В (типа Блекуэлла). Если $\xi(t)$ - неприводимый возвратный полумарковский прочесс (ПМП), то $\forall i, j, \forall h>0$

$$
\lim _{t \rightarrow+\infty} \int_{t}^{t+h} \Phi_{i j}(x) d x=\frac{h}{\mu_{j j}}, \quad i, j=1, \ldots, m
$$

Теорема С (типа Смита). Пусть $g_{j}(t), j=1, \ldots, m$, непосредственно интегрируемая по Риману функиия. Тогда если $\xi(t)$ - неприводимый возвратный ПМП, то

$$
\lim _{t \rightarrow+\infty} \int_{0}^{t} \Phi_{i j}(x) g_{j}(t-x) d x=\frac{1}{\mu_{j j}} \int_{0}^{\infty} g_{j}(x) d x .
$$

В обеих теоремах фигурирует неприводимый невозвратный ПМП $\xi$, порождающий систему $(0.3),(0.4)$ со стохастической матрицей $A$. Через $\mu_{j j}$ обозначено среднее время возврашения в $j$-е состояние. В [5] значения $\mu_{j j}$ не указаны.

Основная новизна результатов настояшей работы по сравнению с теоремами $\mathrm{B}, \mathrm{C}$ и приведенными во введении результатами работ [3], [4] заключается в следуюшем:

a) доказано сушествование пределов $\varphi(+\infty)$ и (6.9) при более слабых ограничениях на $g$ или $u$;

б) найдены компактные формулы для указанных пределов;

в) выведены принципиально новые, структурные, формулы (4.10) и (6.9).

В конце работы укажем на некоторые возможные обобшения результатов настоящей работы.

В условиях критичности (0.4) фигурирует требование $A \in P_{N}$ и $\lambda=1$. Неразложимость матрицы $A$ означает, что система (0.3) не содержит изолированную подсистему. Отказ от этого требования не приводит к принципиальному усложнению задачи. Тогда можно использовать нормальную форму разложимой матрицы $A$ (см. [15]) и соответствуюшую нормальную форму системы (0.3). При этом можно последовательно рассматривать подсистемы, соответствуюшие клеткам нормальной формы, и подсистемы, возникаюшие в результате подстановки решений предыдуших подсистем. Эти решения будут фигурировать в выражениях свободных членов новых подсистем. Каждая из указанных подсистем является либо докритической $(c \lambda<1)$, либо критической. Можно убедиться, что в результате получится решение всей системы, имеюшее степенной рост в $+\infty$.

Теоремы 4.1, 5.1 и 6.1 могут быть использованы при изучении системы (0.3) в надкритическом случае (НKC) $\lambda>1$. С помощью известного простого преобразования НКС легко сводится к критическому случаю. Нетрудно убедиться, что при $\lambda>1$ существует число $p>0$ такое, что $r\left(A_{p}\right)=1$, где $A_{p}=\int_{0}^{\infty} e^{-p x} u(x) d x$. Умножая обе части (0.1) (в матричном случае) на $e^{-p x}$, получаем

$$
\varphi_{p}(x)=g_{p}(x)+\int_{0}^{x} u_{p}(x-t) \varphi_{p}(t) d t .
$$


Индекс $p$ означает умножение функции на $e^{-p x}$, например, $\varphi_{p}(x)=e^{-p x} \varphi(x)$. Нам остается использовать асимптотические свойства решения $\varphi_{p}$ полученного критического уравнения.

Автор выражает благодарность рецензенту настоящей работы за ценную информацию и полезные замечания.

\section{Список литературы}

1. Феллер В. Введение в теорию вероятностей и ее приложения. Т. 2. М.: Мир, 1984.

2. Беллман P., Кук K. Дифференциально-разностные уравнения. М.: Мир, 1967.

3. Цалюк З. Б. Об асимптотике решений уравнения восстановления // Дифференц. уравнения. 1970. Т. 6. №6. С. 1112-1114.

4. Севастьянов Б. А. Теория восстановления // Итоги науки и техники. Теория вероятностей, матем. статистика, теор. кибернетика. Т. 11. М.: ВИНИТИ, 1974. С. 98-128.

5. Королюк В. С., Броди С. М, Турбин А. Ф. Полумарковские процессы и их применение // Итоги науки и техники, Теория вероятностей, матем. статистика, теор. кибернетика. Т. 11. М.: ВИНИТИ, 1974. С. 47-97.

6. Севастьянов Б. А., Чистяков В. П. Уравнениемногомерного восстановления и моменты ветвящихся процессов // Теория вероятностей и ее прим. 1971. Т. 16. № 2. С. 207-217.

7. Цалюк З. Б. Интегралњные уравнения Вольтерра // Итоги науки и техники, матем. анализ. Т. 15. М.: ВИНИТИ, 1977. С. 131-198.

8. Енгибарян Н. Б. Факторизация матриц-функций и нелинейные интегральные уравнения // Изв. АН Арм.ССР. Сер. матем. 1980. Т. 15. № 3. С. 233-244.

9. Енгибарян Н. Б., Арабаджян Л. Г. Системы интегральных уравнений Винера-Хопфа и нелинейные уравнения факторизации // Матем. сб. 1984. Т. 124. № 2. С. 189-216.

10. Арабаджян Л. Г., Енгибарян Н. Б. Уравнения в свертках и нелинейные функциональные уравнения // Итоги науки и техники. Матем. анализ. Т. 22. М.: ВИНИТИ, 1984. C. $175-244$.

11. Арабаджян Л. Г. О системах интегральных уравнений восстановления // Дифференц. уравнения. 1984. Т. 20. №6. С. 1050-1055.

12. Ланкастер П. Теория матриц. М.: Наука, 1978.

13. Геворкян Г. Г., Енгибарян Н. Б. О существовании предела в бесконечности решения уравнения восстановления // Изв. НАН РА. Сер. матем. 1997. Т. 32. № 1. С. 5-20.

14. Енгибарян Н. Б. О нелинейных уравнениях факторизации операторов // Применение методов теории функций и функционального анализа к задачам математической физики. Ереван: Изд-во ЕрГУ, 1982. С. 123-128.

15. Гантмахер Ф. Р. Теория матриц. М.: Наука, 1988.

Бюраканская астрофизическая обсерватория НАН РА П Поступила в редакцию 02.04.1997 и 23.10 .1997 